\title{
Study on New Technology of Electronic and Information Engineering
}

\author{
Gang Wu \\ College of Telegraph, Pan Zhihua University,Pan Zhihua,617000,China \\ email:wugang_d@126.com
}

Keywords: Electronic Information Engineering; New Technology; Research

\begin{abstract}
. electronic and information engineering is a subject of information control and processing with modern technology as the foundation. Electronic information industry is the leading industry of today's social development, It is plays a role of multiplication and amplification on the national defense capability and national economic development, it is also the focus of international competition, Therefore, this paper discussed the new technology of the electronic information engineering from the current situation and the existing problems of the development of electric information engineering, and make the students master the professional courses at the same time, to understand the development of new technology of electronic and information engineering, the purpose is the good foundation for the following development .
\end{abstract}

\section{Introduction}

Electronic and information engineering is a subject of information control and processing with modern technology as the foundation. It mainly studies the acquisition of information, storage of information, transmission of information, detection of information, control of information , processing of information, and equipment of electronic information ,development of related system , design of related system, application of related system and integration technology etc.

Over the years, electronic and information engineering has penetrated into every field of people's production and life, it has been pushing the development of electronic industry and technology, but also it promote the differentiation and integration of industrial chain, to break the boundaries between the industry, it make boundary fuzzy between the industry. At the same time, The new technology of electronic and information engineering is the electronic information industry to break through the traditional technology and mode, realize the base of technology innovation, It is a turning point in the higher stage of the electronic information engineering development [1]. Therefore, it has the certain practical significance to explore the new technology of the system of electronic information engineering.

\section{Present Situation and the Existence Question of Electrical Information Engineering Development}

With the rapid development of electronic information industry, electronic information products of, high-tech, high technology, high efficiency, high quality and other industries combined, the formation of the industrial chain of electronic information engineering, not only to promote the development of electronic information industry to high quality, but also promoted the growth of GDP, However, modern technology of the electronic information engineering is in rapid development at the same time, now also faces some problems, It is mainly reflected in the following aspects: (1) From the undergraduate education, the curriculum system of electronic information engineering and modern electronic technology of rapid development is not synchronized, training plan changes little with the traditional course content, It is mainly based on theory based teaching, practice teaching for less class time, such as "data acquisition technology" (36 hours for theory and experiment of 8 hours), teaching content is based on the concept, sensor, circuit and anti-interference technique etc, Seriously out of line with the industry IT of large scale integrated circuit of current development, In the work, students 
generally feel "they has learned the knowledge not to use in practice, in practice they need knowledge not to learn. (2) From the national level, compared with the developed countries in electronic information technology, the overall level of China's electronic information technology is not high, although some industries have been the introduction of foreign advanced technology, but to a certain extent restricted the development process of the independent innovation of China's electronic information industry. (3) From the development of the industry, electronic information industry belongs to the high-tech industry, its characteristic is the combination of knowledge and technology, the combination of market and products, at present in some industry over reliance on foreign technology, the lack of awareness of innovation, the lack of independent brands and patent technology of the intellectual property rights [2].

\section{The new technology of Electronic Information Engineering}

Architecture of new technology of Electronic information engineering :We Can know from Figure 1, The new technical content of electronic information engineering classification and organic integration based on the training goal of electronic and Information Engineering, make its build around "a whole electronic information and communication system", covering the electronic science ,technology of communication, information science, computer technology and other major disciplines, The purpose is to cultivate innovative talents service with "thick foundation, wide caliber, great capability and high quality" [3] .

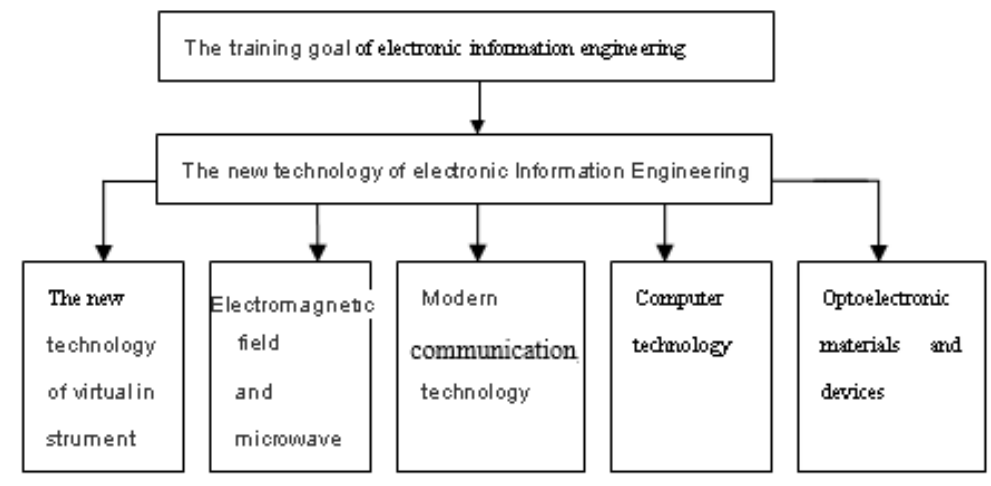

Fig. 1. the Architecture of new technology of electronic Information Engineering

The new technology of virtual instrument: Electronic measuring instrument is the measurement equipment of various electric and non electric, it has experienced several stages of a pointer type, digital, intelligent, virtual instrument etc, With the development of technology, it often require the measurement of many electrical or nonelectric quantity at the same time, to electric and non electric tracking and measuring, the measuring results can comprehensive analysis, to realize the measurement of this function is virtual instrument, which is composed of a personal computer, the function hardware of modularization, control software and application software, Through the operation of the graphical interface of the image and programming language, it can complete the acquisition, analysis, judgment, display, data storage, graphics generation etc. The computer screen as a virtual panel of measuring instrument. not only It contains knob and switch in the virtual panel, but also it contains the menu, data and waveform diagram etc. The function hardware of modularization is inserted in the expansion box, which comprises a signal generator card, frequency meter card, oscilloscope card, logic analyzer card, spectrum analyzer card, IC test card, noise tester card, communication card etc. The communication card is responsible for communication between instrument and PC [4].

Modern communication technology: The traditional communication technology With the combination of computer technology, control technology, digital signal processing technology is The typical symbol of modern communication technology, Modern communication technologies include digital communication technology, program-controlled exchange technology , information transmission technology (computer transmission), communication network 
technology, data communication and data network, broadband IP technology, access network and access technology. because of the most important difference between the modern communication and traditional communication is the characteristic of the modern communication technology with modern computer technology combined. The general trend of the technical development in optical fiber communication as the main body, satellite communication and radio communication as the auxiliary, the broadband, integrated(some call digital), personalized, intelligent communication network technology as the main content and direction of development, goal is to achieve wide bandwidth, large capacity, long distance communication, multiuser, high security, high efficiency, high reliability, high flexibility. If there is no digital, broadband, intelligent and personalized is difficult to achieve; there is no broadband integrated services digital network, it is very difficult to realize intelligent and personalized, the "four modernizations" of modern communication technology is actually the specific content of the "information superhighway", with the development of modern communication technology. Human society has gradually entered the information society [5].

Electromagnetic field and microwave technology: The main research of electromagnetic field and microwave technology is generation of radio frequency signal, radiation of radio frequency signal, propagation of radio frequency signal, scattering of radio frequency signal, receiving and processing of radio frequency signal, the specific performance in daily life: the radio communication, radio, cell phone signal transmission, satellite television, in industry, medical and defense ,it's the specific performance is application of radar, remote sensing, telemetry and telecontrol, geophysical exploration, electronic measurement, electronic warfare, radio astronomy and nondestructive detection etc, especially microwave technology [the frequency range from $\quad 300 \mathrm{MHz}(1 \mathrm{~m})$ to $3000 \mathrm{GHz}(0.1 \mathrm{~mm})]$,Because the microwave is like light, penetrating characteristics, wideband characteristic, scattering characteristic, anti low frequency interference, line of sight propagation characteristics, thermal effect characteristics, information, non ionizing and the uncertainty of distribution parameters, it is widely used in the microwave heating and microwave weapons, with the in-depth study of microwave technology application ,the microwave and its application scope has been expanded to the following aspects: (1)It can be realized on the puffing, drying, deodorization and fresh keeping processing, at present it has been used in cakes, dried beef, potato chips, instant food processing, tea antivirus, drying and so on; (2) in medical treatment, It can cure cancer with radiotherapy and chemical therapy combined; and also can be heated plasma and thawing refrigerated organs by microwave; and microwave surgical knife; (3) In the semiconductor manufacturing process, it has been using microwave plasma technology, etching, sputtering, gas phase deposition, silicon wafer of oxidation; also it can be used for metal, alloy, non metal surface treatment; it used spectral analysis of plasma, it can be detection more than ten kinds of elements. We can know from the advantage of application of microwave technology in all aspects : in the near future, microwave may become a conventional technology in industrial production, environmental protection and other fields [6].

Computer technology: Electronic information engineering is a discipline of modern technology for electronic information control and information processing by the application of computers, With the development of modern computer technology, it is not only a strong impetus to the rapid development of electronic information technology, but also computer theory and technology has infiltrated into the basic and specialized course content of the electronic information engineering , Its relationship with electronic information engineering is increasingly close . which is mainly reflected in the following aspects: 1)the close combination of computer technology and microelectronics technology, the embedded computer system has been developed rapidly, but also makes the embedded system chip SOC (System on Chip) application widely, high performance MCU and dedicated microprocessor chip (such as DSP) has gradually in all kinds of instruments, intelligent control of equipment (including household appliances); (2)With the rapid development of software technology, makes the electronic information into the computer program in the field 
of data operation, data transformation and data processing, the algorithms of computer technology has unique advantages in the electronic information technology. (3) With the rapid development of computer simulation technology, making the design , analysis and simulation of electronic circuit and communication system is more convenient and efficient, appeared a lot of applications software for different levels of EDA (Electronic Design Automation), covering the whole process of electronic design [3].

optoelectronic materials: Electronic information industry is the leading industry of today's social development, It is plays a role of multiplication and amplification on the national defense capability and national economic development, it is also the focus of international competition, and electronic and optoelectronic functional materials is one of the key foundation of electronic information industry, it offer the main function of use for electronic devices and optoelectronic device material through direct effect and the coupling effect of the electric, magnetic, acoustic, elastic, thermal, chemical and biological, The characteristics of its development is: (1) categories, varieties and specifications is particularly much, but each of the purposes. (2) The materials science is an interdisciplinary subject, it involves chemistry, physics, electronics, mechanics etc, It has the characteristics of Polytechnic crossing, (3)The subject is a development of the less mature subject, the development of materials depend greatly on the experiment, the fact and the accumulation of experience [7] .

\section{Conclusion}

Electronic information industry is the leading industry of today's social development, It is plays a role of multiplication and amplification on the national defense capability and national economic development, it is also the focus of international competition. From the above analysis shows that: the new technology of electronic information engineering relates to virtual instrument new technology, modern communication technology, electromagnetic field and microwave technology, computer technology, optoelectronic materials and devices, it may not be fully reflected in the curriculum system construction, it can only be related to introduce the process of teaching in the classroom, or make presentations by lectures, and make the students master the professional courses at the same time, to understand the development of new technology of electronic and information engineering, the purpose is the good foundation for the following development .

\section{References}

[1] Rui Ha. Study on modern technology of electronic and information engineering [J], information system engineering, 2003(11) 16-20

[2]. Qiuhua Wang. The explore on curriculum reform of electronic information engineering of our school [J]. China Science and technology information 2008 (8) 229 -230

[3]Yongshun Liu, Jin Wang. Development trend of Modern electronic measuring instrument [J], Journal of Anyang Normal University, 2008 (2) 56-58

[4]Ruirui Yao, the general trend of modern communication technology development [J],Chinese high-tech enterprises 2012 (026-8

[5]Yan Xu, Qilong,Hou ,Quan Hou . The development and the prospect of modern communication [J], Silicon Valley, 2012 (3) 97-98

[6] Zining Yang. Development and application of microwave technology [J], China information of science and Technology 2006 (18) 142-143

[7]Xiaoyi Dong, Xueshen Lv. Development of optoelectronic devices [J], optoelectronic .laser 1990(1) 46-50 